INPLASY

PROTOCOL

To cite: Tian et al. The

Therapeutic Effects of a Chinese Medicine Formula Huangjing Zanyu capsule for the Treatment of Maleinfertility: A Meta-Analysis of

Randomized Controlled Trials. Inplasy protocol 202230027. doi:

10.37766/inplasy2022.3.0027

Received: 07 March 2022

Published: 07 March 2022

Corresponding author:

Zhaohui Tian

dytianzhaohui@163.com

Author Affiliation:

Nanjing University of Chinese

Medicine

Support: None.

Review Stage at time of this submission: Completed but not published.

Conflicts of interest:

None declared.

\section{The Therapeutic Effects of a Chinese Medicine Formula Huangjing Zanyu capsule for the Treatment of Maleinfertility: A Meta-Analysis of Randomized Controlled Trials}

Tian, ZH¹; Zeng, QQ2; Weng, HW³.

Review question / Objective: The aims of this systematic review is to appraise the efficacy and safety of a traditional chinese medicine formula Huangjing Zanyu capsule for the treatment of Male infertility.

Information sources: We will identify relevant randomized controlled trials from 1970 to November 2021 by a systematic search of PubMed, Web of Science, the Cochrane Library, Chinese BioMedical Literature Database, China NationalKnowledge Infrastructure (CNKI), China Science and Technology Journal database (VIP) and WanfangData. In addition, ongoing trials will be retrieved from the WHO ICTRP Search Portal, the ChineselinicalTrial Register and The Clinical Trials Register.

INPLASY registration number: This protocol was registered with the International Platform of Registered Systematic Review and Meta-Analysis Protocols (INPLASY) on 07 March 2022 and was last updated on 07 March 2022 (registration number INPLASY202230027).

\section{INTRODUCTION}

Review question / Objective: The aims of this systematic review is to appraise the efficacy and safety of a traditional chinese medicine formula Huangjing Zanyu capsule for the treatment of Male infertility.
Condition being studied: Currently, about $15 \%$ of married couples in the world are affected by infertility, among which infertility caused by male factors alone accounts for about $50 \%$.Studies have shown that a chinese medicine formula Huanging Zanyu capsule can increase sperm motility, concentration, and jointly 
improve sperm quality andmate pregnancy rate.

\section{METHODS}

Participant or population: The patients conforming to the diagnostic criteria established in the Guidelines for the diagnosis andtreatment of andrological diseases in China in 2013, Andrology branch, Chinese Medical Association, orother authoritative diagnostic criterias.

Intervention: The included trials met the following stringent intervention criteria. The intervention group was given a single treatment of Huangjing Zanyu capsule for 3 months or 6 months.

Comparator: The control groups had a treatment with placebo or other drugs but not Huangjing Zanyu capsule capsules.Cointerventions were allowed as long as all arms of a trial received the same therapies.

Study designs to be included: We included only randomised trials to assess the beneficial effects of the treatments, and no observational studies will be included.

Eligibility criteria: The included trials met the following stringent intervention criteria. The intervention group was given a single treatment of Huangjing Zanyu capsule for 3 months or 6 months.

Information sources: We will identify relevant randomized controlled trials from 1970 to November 2021 by a systematic search of PubMed,Web of Science, the Cochrane Library, Chinese BioMedical Literature Database, China National Knowledge Infrastructure (CNKI), China Science and Technology Journal database (VIP) and WanfangData. In addition, ongoing trials will be retrieved from the WHO ICTRP Search Portal, the ChineselinicalTrial Register and The Clinical Trials Register.

Main outcome(s): Sperm concentration, sperm motility, sperm morphology.
Quality assessment / Risk of bias analysis: For the risk of bias in the included literature, refer to cochrane Handbook 5 The "bias risk assessment" tool in 1.0.

Strategy of data synthesis: We will identify relevant randomized controlled trials from 1970 to November 2021 by a systematic search of PubMed,Web of Science, the Cochrane Library, Chinese BioMedical Literature Database, China National Knowledge Infrastructure (CNKI), China Science and Technology Journal database (VIP) and WanfangData. In addition, ongoing trials will be retrieved from the WHO ICTRP Search Portal, the ChineselinicalTrial Register and The Clinical Trials Register.

Subgroup analysis: Subgroups will be designed according to the type of clinical study.

Sensitivity analysis: Change the effect model for analysis.

Country(ies) involved: China.

Keywords: Huangjing Zanyu capsule; Male infertility; Randomized controlled trial; Meta analysis.

Contributions of each author:

Author 1 - Zhaohui Tian.

Author 2 - Qingqi Zeng.

Author 3 - Haowei Weng. 